\title{
Urinary Symptoms and Urodynamics Findings in Patients with Friedreich's Ataxia
}

André F. A. Musegante, Priscila Natasja S. Almeida, Raphael Temporão M. Monteiro, Ubirajara Barroso Jr

SARAH Network of Rehabilitation Hospitals (AFAM, PNSA), Salvador; SARAH Network of Rehabilitation Hospitals (RTMM), Brasilia and Bahiana University of Bahia (UBjr), Salvador, BA, Brazil

\section{ABSTRACT}

Purpose: To assess the prevalence of LUTS, urinary tract and urodynamics changes in patients with Friedreich's Ataxia (FA), the most common form of hereditary ataxia.

Materials and Methods: This study evaluated 258 patients with genetically confirmed diagnoses of FA. Of the patients, 158 responded to a questionnaire which assessed their urinary symptoms. Patients with clinical changes underwent renal function examinations, ultrasound, and urodynamic studies (UDS).

Results: The sample analyzed showed that $82 \%$ of the patients complained of LUTS, although only $22 \%$ related the symptoms with quality of life impairment. Twenty eight $(18 \%)$ of them agreed to undergo urodynamic evaluation. Urgency was the most common symptom. The exam was normal in $4(14 \%)$ and detrusor underactivity was the most common finding. 14\% (4 patients) presented with dilatation of the upper urinary tract at ultrasound scans. None of them had creatinine alterations.

Conclusions: LUTS was found in a large percentage of patients with FA, but only a few related it to their quality of life impairment. Although creatinine levels was normal in this sample, some patients may show upper urinary tract abnormalities, with deserves close observation and proper care.

\section{ARTICLE INFO}

\author{
Key words: \\ Friedreich Ataxia; \\ Urodynamics; Lower \\ Urinary Tract Symptoms; \\ Urinary Bladder; Urination \\ Disorders
}

Int Braz J Urol. 2013; 39: 867-74

Submitted for publication:

October 10, 2012

Accepted after revision:

July 24, 2013

\section{INTRODUCTION}

Freidreich's ataxia (FA) is the most common form of hereditary ataxia, with an estimated prevalence of 2 to 3 people per 100,000 (1). It consists of a neurodegenerative disease associated with a dynamic mutation of the trinucleotide guanine adenine adenine (GAA) in the first intron of the gene $\mathrm{x} 25$, located on the long arm of chromosome 9 (9q11). The trinucleotide repeat expansion causes a reduction in the synthesis of the protein frataxin, and the lowered frataxin levels in the mitochondria cause oxidative damage and progressive neuronal degeneration (2). The clinical diagnosis is confirmed with a genetic test that demonstrates the GAA repeat expansion. In a normal individual, the GAA sequence is repeated 7 to 22 times. Those who suffer from FA can have between 200 and over 1,000 repetitions of the GAA sequence. In general, more repetitions of the GAA trinucleotide signify an earlier onset of and more severe case of FA (3).

It is known that LUTS can lead to a worsened quality of life, but its most severe effect is renal function impairment. The objective of this study was to evaluate clinical and urodynamic changes in the lower urinary tract (LUT) of patients with FA. In contrast with previously published studies, 
we worked with a larger number of patients, all of whom had previously undergone a molecular evaluation with regards to their FA.

\section{MATERIALS AND METHODS}

All 258 patients with genetically confirmed cases of FA using the Polymerase chain reaction (PCR) method were evaluated. Any patients without genetic confirmation, with associated neurological disorders or cognitive impairment, or with surgical history in the LUT were excluded. A telephone interview was conducted to gather information about LUTS. In the absence of a validated questionnaire for neurogenic bladder, one was created by the authors specifically for this paper. (Table-1). Patients who underwent clinical changes in the LUT were invited to continue participating in the research of the urinary tract with renal function tests, kidney and urinary tract ultrasounds, and UDS. During the UDS (Medtronic Duet, Minneapolis, Minnesota, USA) uroflowmetry was initially carried out; followed by cystometry and pressure flow studies. An anal electrode was used to record the electromyographic data of the external urethral sphincter and perineal muscles. Changes in the detrusor contractions were defined as: underactivity (contraction of reduced strength and/or duration, resulting in prolonged bladder emptying and/or a failure to achieve complete bladder emptying within a normal time span); overactivity (involuntary detrusor contractions during the filling phase which may be spontaneous or provoked); acontractile (contraction cannot be demonstrated during urodynamic studies) (4). Detrusor sphincter dyssynergia was considered as detrusor contraction concurrently with an involuntary contraction of the urethral and/or periurethral striated muscle (4). Bladder capacity was considered reduced when it was less than $350 \mathrm{~mL}$ (4). Post voiding residue was considered insignificant to $100 \mathrm{~mL}$ (5). Normal flow depends on the age and gender: Males $<40 \mathrm{yr}>$ $22 \mathrm{~mL} / \mathrm{s}, 40-60 \mathrm{yr}>18 \mathrm{~mL} / \mathrm{s}$ e $>60 \mathrm{yr}>13 \mathrm{~mL} / \mathrm{s}$. Females < $50 \mathrm{yr}>25 \mathrm{~mL} / \mathrm{s}$ e $>50 \mathrm{yr}>18 \mathrm{~mL} / \mathrm{s}$ (6). The study also followed ultrasound results and urinary levels of creatinine and urea. The results shown correspond to mean \pm standard deviation.

Table 1 - Questionnaire of urinary symptoms.

1. a. Do you believe that the number of times you void per day has increased?

1. b. If yes, do you void more than 6 times during the day?

2. a. Do you wake up at night to void?

2. b. If yes, do you void more than 3 times during the night?

3. Do you lose urine on clothing during the day?

4. Do you lose urine on clothing without feeling it?

5. Do you feel an urgency to urinate?

6. Do you have difficulty voiding?

7. Is your urinary stream interrupted during urination?

8. Do you feel an urge to urinate?

9. Does your bladder feel empty when you are finished urinating?

10. Have your urinary symptoms interfered with your quality of life? 
We applied the chi-square test for the relationship between post void residual urine and DU/ detrusor acontractility (DA), detrusor-sphincter dyssynergia (DSD) and alteration in detrusor contraction/ FIM scale. Fisher's exact test was used for pyramidal tract changes as well as changes in the detrusor contractions/DSD, renal dilatation/ FIM scale, residue/FIM scale and DO and urgency. This study was approved by the Institutional Review Board. All patients have signed an informed consent.

\section{RESULTS}

Out of the 258 medical records, 45 (17\%) had out of date telephones, 7 patients (3\%) refused, and 206 patients (80\%) agreed to participate in the study. Of those who agreed to participate in the study, 48 (23\%) failed to respond so we have analyzed the results on 158 patient samples. Data on the neurological symptoms of patients who participated in the urological research are shown in Figure-1.

The primary complaints of 129 of the patients were related to storage symptoms. Frequency was cited by the majority; $63 \%$ of the patients voided more than 6 times per day, 46\% were waking up at night to void, and 36\% had urinary incontinence. Despite the clinical findings, only $35(22 \%)$ believed that their LUTS interfered with their quality of life (Table-2).

Out of the 129 patients who showed LUT dysfunction, 28 (7 men and 21 women) agreed to undergo urodynamic diagnostic testing. The mean age of this subgroup was $32( \pm 11.2)$. The mean age of the patients during the onset of the disease was $16( \pm$ 7) years and the average time that passed between the disease onset and the study was $20( \pm 9)$ years.

Clinically, the most frequently reported symptom on this sub-group of subjects was urgency, present in 21 (75\%) of patients. Urinary incontinence was seen in 17 (61\%), urinary frequency in 11 (39\%), effort to urinate in 9 (32\%), nocturia in 7 (25\%), and incomplete bladder emptying in 7 (25\%) (Table-3).

All 28 patients underwent UDS, which showed up normal in 4 (14\%) patients. Changes in detrusor contraction were observed in half the patients, with DU being the most common abnormality. Of the patients with D0, only one had detrusor pressure greater than $40 \mathrm{cmH}_{2} \mathrm{O}\left(53 \mathrm{cmH}_{2} 0\right)$. There was no change in bladder compliance in any patient.

Bladder sensitivity was altered in 17 (60.7\%) patients; diminished in $14(50 \%)$ and increased in $3(10.7 \%)$. Bladder capacity was below what is considered normal in only $4(14 \%)$ patients. Urine flow was diminished in 14 (50\%) of patients, taking gender and age into consideration. In 11 (39\%) patients the flow was below $10 \mathrm{~mL} / \mathrm{s}$, and 3 patients with DA had no voiding. The residual volume after voiding was greater than $100 \mathrm{~mL}$ in 11 (39\%) patients, 8 patients had changes in detrusor contractions (acontractility and underactivity) and 3 had normal detrusor contractions. DSD was

Figure 1 - Frequency of non-urological features in 28 patients with LUTS.

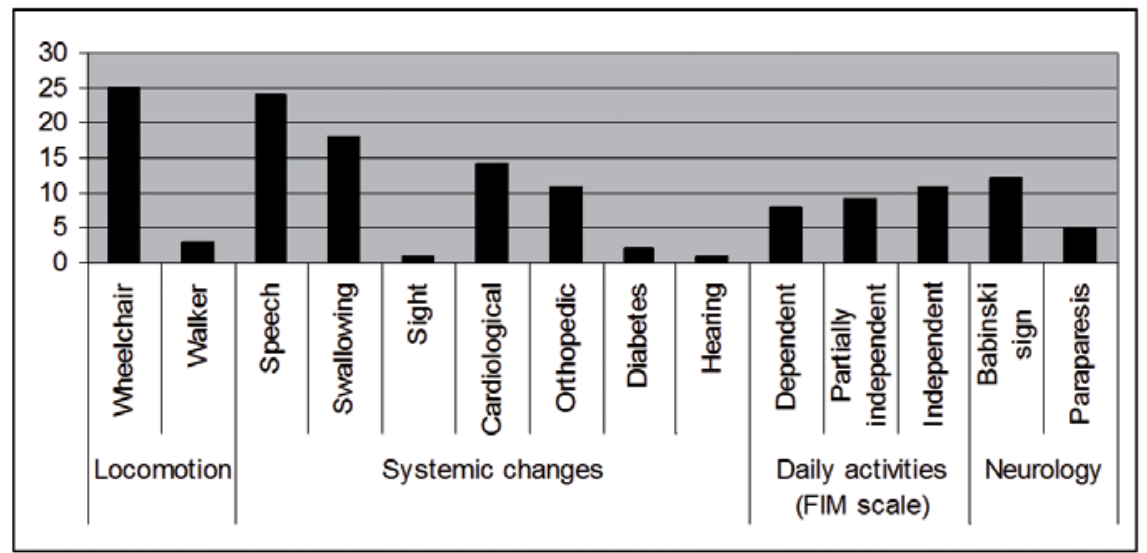


Table 2 - Frequency of each question in the questionnaire.

\begin{tabular}{lcc}
\hline Questions & $\begin{array}{c}\text { Frequency } \\
\text { (N=158) }\end{array}$ & $\%$ \\
\hline 1.a. Do you believe that then number of times you void per day has increased? & 65 & $41 \%$ \\
1.b. If yes, do you void more than 6 times during the day? & $41(\mathrm{~N} 65)^{\star}$ & $63 \%$ \\
2.a. Do you wake up at night to void? & 73 & $46 \%$ \\
2.b. If yes, do you void more than 3 times during the night? & $11(\mathrm{~N} 73)^{*}$ & $15 \%$ \\
3. Do you lose urine on clothing during the day? & 57 & $36 \%$ \\
4. Do you lose urine on clothing without feeling it? & 27 & $17 \%$ \\
5. Do you feel an urgency to urinate? & 23 & $14 \%$ \\
6. Do you have difficulty voiding? & 29 & $18 \%$ \\
7. Is your urinary stream interrupted during urination? & 45 & $29 \%$ \\
8. Do you feel an urge to urinate? & 3 & $2 \%$ \\
9. Does your bladder feel empty when you are finished urinating? & 40 & $27 \%$ \\
10. Have your urinary symptoms interfered with your quality of life? & 35 & $22 \%$ \\
\hline
\end{tabular}

* The frequency ( $\mathrm{n}$ ) in the questions 1.b and 2.b refers to those who answered "yes" in the previous question

Table 3 - Frequency of urinary symptoms in the 28 patients who participated in the urodynamic study.

\begin{tabular}{lcc}
\hline Urinary Symptoms & $\begin{array}{c}\text { Frequency } \\
(\mathrm{N}=28)\end{array}$ & $\%$ \\
\hline Urgency & 21 & $75 \%$ \\
Incontinence & 17 & $61 \%$ \\
Increased Frequency & 11 & $39 \%$ \\
Effort to Urinate & 9 & $32 \%$ \\
Post Void Residual Urine & 7 & $25 \%$ \\
Altered stream & 6 & $21 \%$ \\
Nocturia & 7 & $25 \%$ \\
\hline
\end{tabular}

found in $8(28.5 \%)$ patients, 6 women and 2 men (Table-4).

Creatinine and urea levels were not altered in any of the patients. Ultrasounds showed mild to moderate dilation of the upper urinary tract of 4 (14.3\%) patients. In 7 (25\%), the bladder was irregularly shaped and had a thickened wall.
The relationship between post-void residual urine and DU/DA and DSD was significant $(\mathrm{p}=0.0004)$. The relationship between pyramidal tract changes and changes in detrusor contractions/DSD, alteration in detrusor contraction/ FIM scale, renal dilatation/ FIM scale and residue/ FIM scale FIM was insignificant $(p=0.5, p=0.12, p$ 
Table 4 - Urodynamics findings in 28 patients with FA.

\begin{tabular}{|c|c|c|c|}
\hline Urodynamics Data & & $\begin{array}{c}\text { Frequency } \\
(N=28)\end{array}$ & $\%$ \\
\hline \multicolumn{4}{|l|}{ Detrusor } \\
\hline & Overactivity & 5 & 17.9 \\
\hline & Underactivity & 6 & 21.4 \\
\hline & Acontractility & 3 & 10.7 \\
\hline & Normal & 14 & 50.0 \\
\hline \multicolumn{4}{|l|}{ Sensitivity } \\
\hline & Increased & 3 & 10.7 \\
\hline & Decreased & 14 & 50.0 \\
\hline & Normal & 11 & 39.3 \\
\hline \multicolumn{4}{|c|}{ Post Void Residual Urine } \\
\hline & Increased & 11 & 39 \\
\hline & Insignificant & 17 & 61 \\
\hline \multicolumn{4}{|l|}{ Flow } \\
\hline & Normal & 17 & 61 \\
\hline & Altered & 11 & 39 \\
\hline \multicolumn{4}{|l|}{ Bladder Capacity } \\
\hline & Normal & 24 & 86 \\
\hline & Decreased & 4 & 14 \\
\hline \multicolumn{4}{|l|}{ Compliance } \\
\hline & Normal & 28 & 100.0 \\
\hline & Decreased & 0 & 0.0 \\
\hline \multicolumn{4}{|l|}{ Dyssynergia } \\
\hline & Present & 8 & 28.6 \\
\hline & Absent & 20 & 71.4 \\
\hline
\end{tabular}

$=1.0$ and $\mathrm{p}=0.7$ respectively). Urgency and DO were unrelated $(p=0.6)$.

\section{DISCUSSION}

Patients initially enter the clinic between the ages of 8 and 15 and are diagnosed with gait ataxia. After 10 to 15 years, this ataxia develops and the patients are unable to walk. The life expectancy after the beginning of the disease is estimated to be between 35 and 40 years. The first sign is the ataxia progressively affecting walking and posture, and later it begins to affect arm movement. Approximately 50\% of these patients have skeletal deformities (scoliosis, pes cavus, and equinovarus). Oculomotor disorders, such asfixa- 
tion, nystagmus and reduction of the vestibulo-ocular reflex, point to cerebellar dysfunction. Cardiac diseases appear in $60 \%$ of patients with FA, the best known of which is concentric hypertrophic cardiomyopathy. Diabetes mellitus is present in 10 to $30 \%$ of patients (7). Lower urinary tract dysfunction has been reported in several ataxias, but has not been studied further $(2,8)$. FA is estimated to have around 50\% of LUTS, but there are a limited number of studies on the subject, no specific questionnaires, and the studies are not always confirmed by molecular analysis (8).

There are few studies in the literature on clinical and urodynamic findings of patients with FA, and the studies that do exist contain data from patients without molecular confirmation and with other types of ataxia $(2,8)$. Therefore, it might be impossible to know whether the findings truly correlate to this particular group.

This study showed that $82 \%$ of patients with FA suffer from at least one symptom described in questions 1-9. If questions 3 and 4 are the only ones considered (relating to incontinence), the prevalence of these symptoms among patients with FA decreases to 40\% $(\mathrm{N}=62)$. Previously published studies show this type of patient presents around $50 \%$ of these urinary symptoms $(2,8,9)$ using the International Prostate Symptoms Score (IPSS), but 56\% of the patients are female (8). There is no significant difference in the mean ages of patients with FA or in the mean time of the disease evolution between our study and previously published literature that accounts for this discrepancy. Unlike our study, however, previous articles neglected to report certain clinical neurological features, including the use of wheelchairs and the patients' degree of independence, which makes it difficult to evaluate the severity of the patients' FA and comparison between the samples (2,8-10). Figure-1 shows that less than 43\% of patient samples have an impairment in the pyramidal tract or in other systems, which shows the evolutionary state of the disease. It is also noteworthy that the cited studies did not genetically confirm the disease, which would serve to ratify the clinical diagnosis (8).

Urgency was predominantly seen among LUTS symptoms, which is in accordance with the literature. Urinary incontinence was present in $61 \%$ of our patients, which contrasts with the 27.6\% found by Diez Rodriguez et al. (8). Note that the IPSS does not question patients about incontinence. There are a few possible explanations for this discrepancy: the difference in the population studied (our patients were studied genetically); patients in our study had not received any treatment; or perhaps neurological impairment was more pronounced in our patients (8). Other symptoms such as nocturia, frequency, feeling of incomplete bladder emptying, and effort to urinate are in accordance with other published works. Based on this variety of findings, we agree with Rodriguez et al. (8) who argued that the variety of symptoms and urodynamic findings in patients with ataxia is due to the multi-factorial damaging potential to the central nervous system during the evolution of FA, considering that all segments of the nervous system (posterior cord, spinocerebellar pathways, pyramidal tracts, spinal ganglia, cerebellum and peripheral nerves) have the potential to be affected. A supra sacral legion could cause damage in the reflex arc and a dyssynergia voiding pattern (2).

In published literature, the most predominant urodynamic finding is DO, ranging from $22 \%$ to $61 \%(2,10,11)$. Our series shows DO in $17.9 \%$ of cases. DU was seen in $21.4 \%$ of patients and DA in $10.7 \%$. Previous literature shows the rate of DA to vary between $23.5 \%$ and $26.7 \%(2,12)$. It is possible that the progression of the disease probably affects the neurophysiologic pathways of the micturition. The presence of DSD (28.5\%) in this study does not differ from the literature (20\% to $37 \%$ ) $(2,10)$ and once again the neurological findings seem to agree with our urological data, because $43 \%$ of our sample has changes in the pyramidal tract.

No changes were found in the renal function of our patients. This data is consistent with the low pressure reached during DO and without any change in compliance. Renal dilatation was found in 4 patients, 2 with changes in bladder sensitivity, 1 with hypocontractility and 1 with acontratility, the latter two being dependent on the FIM scale. All patients were referred for evaluation and treatment, with the latter two initiating 
intermittent catheterization cleaning. Statistically we can not associate renal dilation with neurological worsening (change in FIM scale).

Regarding the clinical-urodynamic correlation we can state that the complaint of urgency does not seem to represent D0, as there were 21 patients with urgency and only 5 with DO. Four out of the $21(19 \%)$ patients who complained of urgency also had DO. The high level of post voiding residual urine seems to demonstrate more precisely the presence of DSD and/or DU/DA, because out of 11 (39\%) patients with high levels of post-void residual, 10 (91\%) had some of these diagnoses, however we found no association between residual and neurological worsening (change of FIM scale). The presence of a lesion in the pyramidal tract seems to be related to changes in sphincter and/or detrusor contractions, as only 4 (40\%) patients with pyramidal changes did not show dyssynergia and/or changes in bladder contraction (overactivity, acontractile, and underactivity). In the literature, only Caraceni demonstrated a statistically significant correlation between pyramidal lesions and D0 and DSD (9). We do not find this relation to be significant.

We used the alteration in the FIM scale to represent patients with more severe neurological impairment, and thus tried to show that these patients have greater urological disorders, however we found no significance. We recognize that there was a low rate of attendance for the completion of the exams. Perhaps the importance of urinary symptoms is reduced before a neurological condition is prominent. With this, we have completed the investigation, especially in patients less affected by the disease. The lack of urodynamic parameters defined in previously published work is another factor that can change the comparison of our findings. Therefore, we know that many present symptoms, but few report them, and that these should always be investigated since changes in the upper and lower urinary tracts were found.

\section{CONCLUSIONS}

LUT dysfunction was found in a large percentage of patients with FA, but only a few related it to their quality of life impairment (22\%).
Urgency was the most prevalent symptom (75\%), and DO was the second most common urodynamic finding (17.9\%). Urgency was associated with D0 in only $14 \%$ of patients. Although creatinine levels were normal in this sample, some patients may show upper urinary tract abnormalities, which deserve close observation and proper care.

\section{ABBREVIATIONS}

$\mathrm{DA}=$ Detrusor acontractility

DO $=$ Detrusor overactivity

$\mathrm{DU}=$ Detrusor underactivity

DSD = Detrusor-sphincter dyssynergia

FA $=$ Freidreich's ataxia

GAA = Guanine adenine adenine

IPSS = International Prostate Symptoms Score

LUT $=$ lower urinary tract

LUTS = lower urinary tract symptoms

PCR $=$ Polymerase chain reaction

UDS $=$ Urodynamic studies

\section{CONFLICT OF INTEREST}

None declared.

\section{REFERENCES}

1. Jankovic JJ, Tolosa E: Parkinson's disease and movement disorders. Philadelphia, Lippincott Williams \& Wilkins. 2002.

2. Diez Rodríguez JM, Salinas Casado J, Fernández del Busto E, Mora Durbán M, Mañas Pelillo A, Muñoz Delgado B, et al.: Clinico-urodynamic correlation in the hereditary ataxias. Arch Esp Urol. 2003; 56: 915-25.

3. La Pean A, Jeffries N, Grow C, Ravina B, Di Prospero NA: Predictors of progression in patients with Friedreich ataxia. Mov Disord. 2008 30; 23: 2026-32.

4. Abrams P, Cardozo L, Fall M, Griffiths D, Rosier P, Ulmsten $U$, et al.: The standardisation of terminology of lower urinary tract function: report from the Standardisation Subcommittee of theInternational Continence Society. Neurourol Urodyn. 2002; 21: 167-78.

5. Griffiths DJ, Harrison G, Moore K, McCracken P: Variability of post-void residual urine volume in the elderly. Urol Res. 1996; 24: 23-6. 
6. Jørgensen JB, Jensen KM: Uroflowmetry. Urol Clin North Am. 1996; 23: 237-42.

7. Delatycki MB, Paris DB, Gardner RJ, Nicholson GA, Nassif $\mathrm{N}$, Storey $\mathrm{E}$, et al.: Clinical and genetic study of Friedreich ataxia in an Australian population. Am J Med Genet. 1999; 87: 168-74.

8. Díez Rodríguez JM, Salinas Casado J, Fernández del Busto E, Navarro Sebastián J, Mora Durbán M, Mañas Pelillo A, et al.: Urogenital alterations in hereditary and sporadic neurodegenerative ataxias. Neurologia. 2005; 20: 17-23.

9. Caraceni E, Leone G, Ardito S: Neurogenic bladder dysfunction in hereditary Friedreich's ataxia. Urodinamica 1992; 2: 177-9.

10. Vezina JG, Bouchard JP, Bouchard R: Urodynamic evaluation of patients with hereditary ataxias. Can J Neurol Sci. 1982; 9: 127-9.
11. Nardulli R, Monitillo V, Losavio E, Fiore P, Nicolardi G, Megna G: Urodynamic evaluation of 12 ataxic subjects: neurophysiopathologic considerations. Funct Neurol. 1992; 7: 223-5.

12. Leach GE, Farsaii A, Kark P, Raz S: Urodynamic manifestations of cerebellar ataxia. J Urol. 1982; 128: 348-50.

Correspondence address: André Ferraz de Arruda Musegante, MD Urologist of SARAH Network of Rehabilitation Hospitals, Av. Tancredo Neves, 2782, Caminho das Árvores, Salvador, Bahia, 41820-900, Brazil Fax: +55 71 3206-3333 E-mail: afmusegante@uol.com.br 